\title{
BOUNDS FOR DETERMINANTS WITH POSITIVE DIAGONALS
}

\author{
BY \\ EMILIE V. HAYNSWORTH(')
}

1. Introduction. In this paper upper and lower bounds are found for the determinant of a real, $n \times n$ matrix $A=\left(a_{i j}\right)$, with positive diagonal elements satisfying

$$
a_{i i} \geqq \sum_{j \neq i}\left|a_{i j}\right|, \quad i=1,2, \cdots, n,
$$

and a lower bound is found for determinants whose elements satisfy

$$
a_{i i} \geqq n A_{i}^{+}-\sum_{j \neq i} a_{i j}, \quad i=1,2, \cdots, n,
$$

where

$$
A_{i}^{+}=\frac{1}{2}\left(\max _{j \neq i} a_{i j}+\left|\max _{j \neq i} a_{i j}\right|\right) .
$$

G. B. Price [6], A. Ostrowski [4] and [5], J. L. Brenner [1] and [2], and $\mathrm{H}$. Schneider [7] have given lower and upper bounds for the absolute value of determinants satisfying more general conditions than (1). However, the following theorem, proved in $\$ 2$, is not implied by any of the above results:

THEOREM 1. If $A=\left(a_{i j}\right)$ has elements satisfying (1), it is possible to define $L_{i}$ and $R_{i}$ such that

$$
\begin{aligned}
a_{i i} & =L_{i}+R_{i}, \\
L_{i} & \geqq \sum_{j<i}\left|a_{i j}\right|, \\
R_{i} & \geqq \sum_{j>i}\left|a_{i j}\right| .
\end{aligned} \quad(i=1, \cdots, n) .
$$

Then, for any choice of $L_{i}$ and $R_{i}$ satisfying (3),

$$
\sum_{k=0}^{n}\left(\prod_{i=1}^{k} L_{i} \prod_{i=k+1}^{n} R_{i}\right) \leqq \operatorname{det} A \leqq \sum_{k=0}^{n} \prod_{i=1}^{k-1}\left(L_{i}+2 R_{i}\right) L_{k} \prod_{i=k+1}^{n} R_{i}
$$

where an empty product is defined to be 1.

Presented to the Society, January 28, 1960; received by the editors November 2, 1959.

(1) The author is presently at Auburn University, Auburn, Alabama. 
Ostrowski [5] has shown that if $A$ satisfies (2) then $\operatorname{det} A \geqq 0$. In $\S 3$ we prove

Theorem 2. If $A$ satisfies (2), then

$$
\operatorname{det} A \geqq \prod_{i=1}^{n}\left(\sum_{j=1}^{n} a_{i j}-n A_{i}^{+}\right) .
$$

In proving this we prove a result which may be used to improve any bound depending only on the nondiagonal elements in the row, when the diagonal elements are positive.

2. Proof of Theorem 1. To prove Theorem 1 we need the following bound given by Price [6]:

If (1) holds, then

$$
\prod_{i=1}^{n}\left(a_{i i}-r_{i}\right) \leqq \operatorname{det} A \leqq \prod_{i=1}^{n}\left(a_{i i}+r_{i}\right)
$$

where $r_{i}=\sum_{j>i}\left|a_{i j}\right|$.

We proceed by induction on $n$. Let $D_{n}$ represent $\operatorname{det} A$, when $A$ is of order $n$, and suppose the elements of $A$ satisfy (3).

1. For $n=2$,

$$
D_{2}=\left|\begin{array}{cc}
L_{1}+R_{1} & a_{12} \\
a_{21} & L_{2}+R_{2}
\end{array}\right| .
$$

Expanding $D_{2}$ by the diagonal elements,

$$
D_{2}=\left|\begin{array}{cc}
L_{1} & a_{12} \\
0 & L_{2}
\end{array}\right|+\left|\begin{array}{cc}
L_{1} & 0 \\
0 & R_{2}
\end{array}\right|+\left|\begin{array}{cc}
R_{1} & 0 \\
a_{21} & R_{2}
\end{array}\right|+\left|\begin{array}{cc}
R_{1} & a_{12} \\
a_{21} & L_{2}
\end{array}\right| .
$$

Therefore

$$
L_{1} L_{2}+L_{1} R_{2}+R_{1} R_{2} \leqq D_{2} \leqq R_{1} R_{2}+L_{1} R_{2}+\left(L_{1}+2 R_{1}\right) L_{2},
$$

since

$$
0 \leqq\left|\begin{array}{cc}
R_{1} & a_{12} \\
a_{21} & L_{2}
\end{array}\right| \leqq\left(R_{1}+a_{12}\right) L_{2}<2 R_{1} L_{2}
$$

by (3) and (5).

2. Assume that for any matrix of order $n-1$ with elements satisfying (3),

$$
\sum_{k=0}^{n-1}\left(\prod_{i=1}^{k} L_{i} \prod_{i=k+1}^{n-1} R_{i}\right) \leqq D_{n-1} \leqq \sum_{k=0}^{n-1}\left(\prod_{i=1}^{k-1}\left(L_{i}+2 R_{i}\right) L_{k} \prod_{i=k+1}^{n-1} R_{i}\right) .
$$

If $D_{n}=\operatorname{det} A$, where $A=\left(a_{i j}\right), i, j=1, \cdots, n$, and the elements $a_{i j}$ satisfy (3), partition $D_{n}$ as follows: 


$$
D_{n}=\left|\begin{array}{cc}
A_{1} & a_{2} \\
a_{3} & L_{n}+R_{n}
\end{array}\right|
$$

where $A_{1}=\left(a_{i j}\right), i, j=1, \cdots, n-1 ; a_{2}$ is the column vector with components $a_{i n}, i=1, \cdots, n-1 ; a_{3}$ is the row vector with components $a_{n j}, j=1, \cdots$, $n-1$; and, as in (3),

$$
\begin{aligned}
L_{n}+R_{n} & =a_{n n}, \\
L_{n} & \geqq \sum_{j=1}^{n-1}\left|a_{n j}\right|, \\
R_{n} & \geqq 0 .
\end{aligned}
$$

Then we can write $D_{n}$ as the sum of two determinants, i.e.,

$$
D_{n}=\Delta+R_{n} \operatorname{det} A_{1}
$$

where

$$
\Delta=\left|\begin{array}{cc}
A_{1} & a_{2} \\
a_{3} & L_{n}
\end{array}\right| .
$$

But the elements of $\Delta$ satisfy (1), hence, by (3) and (5),

and

$$
\begin{gathered}
\Delta \geqq \prod_{i=1}^{n}\left(a_{i i}-r_{i}\right) \geqq \prod_{i=1}^{n}\left(a_{i i}-R_{i}\right)=\prod_{i=1}^{n} L_{i}, \\
\Delta \leqq L_{n} \prod_{i=1}^{n-1}\left(a_{i i}+r_{i}\right) \leqq L_{n} \prod_{i=1}^{n-1}\left(L_{i}+2 R_{i}\right) .
\end{gathered}
$$

Also, by the inductive assumption, since $A_{1}$ is of order $n-1$, and, by (3),

$$
R_{i} \geqq \sum_{j=i+1}^{n}\left|a_{i j}\right| \geqq \sum_{j=i+1}^{n-1}\left|a_{i j}\right|
$$

we have, using (6), (7) and (8),

$$
D_{n} \geqq \prod_{i=1}^{n} L_{i}+R_{n} \sum_{k=0}^{n-1}\left(\prod_{i=1}^{k} L_{i} \prod_{i=k+1}^{n-1} R_{i}\right)=\sum_{k=0}^{n}\left(\prod_{i=1}^{k} L_{i} \prod_{i=k+1}^{n} R_{i}\right)
$$

and

$$
\begin{aligned}
D_{n} & \leqq L_{n} \prod_{i=1}^{n-1}\left(L_{i}+2 R_{i}\right)+R_{n} \sum_{k=0}^{n-1}\left(\prod_{i=1}^{k-1}\left(L_{i}+2 R_{i}\right) L_{k} \prod_{i=k+1}^{n-1} R_{i}\right) \\
& =\sum_{k=0}^{n}\left(\prod_{i=1}^{k-1}\left(L_{i}+2 R_{i}\right) L_{k} \prod_{i=k+1}^{n} R_{i}\right)
\end{aligned}
$$


3. Proof of Theorem 2. Suppose we have a lower bound for the determinant of $A$ which holds when the diagonal elements are individually bounded from below. That is, suppose

$$
a_{i i} \geqq c_{i}(A) \geqq 0, \quad i=1,2, \cdots n,
$$

implies

$$
\operatorname{det} A \geqq m(A) \geqq 0,
$$

where $c_{i}(A)$ is a function of the nondiagonal elements in the $i$ th row, with $c_{i}(A)=0$ if $a_{i j}=0$ for all $j \neq i$, and $m(A)$ is a function of all the nondiagonal elements of $A$.

Let $D=\operatorname{diag}\left(d_{1}, d_{2}, \cdots, d_{n}\right)$ with $d_{i} \geqq 0$. We shall show that

$$
\operatorname{det}(A+D) \geqq m(A)+\prod_{i=1}^{n} d_{i} .
$$

It is this result which we will use to improve Ostrowski's lower bound for determinants satisfying (2).

Since $d_{i i} \geqq c_{i}(D)=0$ for all $i=1,2, \cdots, n,(10)$ is a direct consequence of the following theorem which we proved in [3]:

If two matrices $A$ and $B$ of the same order satisfy the same row hypothesis (i.e., (9)) which is, in turn, sufficient to prove $\operatorname{det} A \geqq 0$ and $\operatorname{det} B \geqq 0$, then $\operatorname{det}(A+B) \geqq \operatorname{det} A+\operatorname{det} B$.

A determinant whose elements satisfy (2) does not necessarily have a dominant diagonal unless $A_{i}^{+}=0(i=1, \cdots, n)$. Ostrowski's result shows, however, that such determinants are never negative, and, as a corollary he proved that, if $\lambda$ is any root of $A$,

$$
\lambda \geqq \mu,
$$

where

$$
\mu=\min _{i}\left(\sum_{j=1}^{n} a_{i j}-n A_{i}^{+}\right) .
$$

Since the determinant is the product of the roots, this would imply

$$
\operatorname{det} A \geqq \mu^{n} \text {. }
$$

This bound is, in general, less than that given by Theorem 2 .

If $A$ satisfies (2), let

$$
\begin{aligned}
& b_{i j}=a_{i j}, \\
& b_{i i}=n A_{i}^{+}-\sum_{j \neq i} a_{i j} .
\end{aligned}
$$

Then $B_{i}^{+}=A_{i}^{+}$, and $B$ satisfies (2). Thus $\operatorname{det} B \geqq 0$.

Let 


$$
d_{i}=a_{i i}-b_{i i}=\sum_{j=1}^{n} a_{i j}-n A_{i}^{+}, \quad i=1,2, \cdots, n,
$$

and set $D=\operatorname{diag}\left(d_{1}, d_{2}, \cdots, d_{n}\right)$. Since (2) implies $a_{i i} \geqq b_{i i}$ we have $d_{i} \geqq 0$ for each $i=1,2, \cdots, n$. Then (10) implies

$$
\operatorname{det} A=\operatorname{det}(B+D) \geqq \prod_{i=1}^{n} d_{i}
$$

which proves Theorem 2 .

\section{REFERENCES}

1. J. L. Brenner, $A$ bound for a determinant with dominant main diagonal, Proc. Amer. Math. Soc. vol. 5 (1954) pp. 631-634.

2. - Bounds for determinants. II, Proc. Amer. Math. Soc. vol. 8 (1957) pp. 532-534.

3. E. Haynsworth, Note on bounds for certain determinants, Duke Math. J. vol. 24 (1957) pp. 313-320.

4. A. Ostrowski, Note on bounds for determinants with dominant principal diagonal, Proc. Amer. Math. Soc. vol. 3 (1952) pp. 26-30.

5. - Note on bounds for some determinants, Duke Math. J. vol. 22 (1955) pp. 95-102.

6. G. B. Price, Determinants with dominant principal diagonal, Proc. Amer. Math. Soc. vol. 2 (1951) pp. 497-502.

7. H. Schneider, An inequality for latent roots applied to determinants with dominant principal diagonal, J. London Math. Soc. vol. 28 (1953) pp. 8-20.

National Bureau of Standards,

Washington, D. C. 